\title{
Dose Distribution of Electrons from Gold Nanoparticles by Proton Beam Irradiation
}

\author{
Jihun Kwon', Kenneth Sutherland2, Takayuki Hashimoto3, Hiroyuki Date ${ }^{4}$ \\ ${ }^{1}$ Graduate School of Health Sciences, Hokkaido University, Sapporo, Japan \\ ${ }^{2}$ Department of Medical Physics, Hokkaido University Graduate School of Medicine, Sapporo, Japan \\ ${ }^{3}$ Department of Radiology, Hokkaido University Graduate School of Medicine, Sapporo, Japan \\ ${ }^{4}$ Faculty of Health Sciences, Hokkaido University, Sapporo, Japan \\ Email: date@hs.hokudai.ac.jp
}

Received 29 December 2014; accepted 20 January 2015; published 27 January 2015

Copyright (C) 2015 by authors and Scientific Research Publishing Inc.

This work is licensed under the Creative Commons Attribution International License (CC BY). http://creativecommons.org/licenses/by/4.0/

c) (i) Open Access

\begin{abstract}
Purpose: In radiation therapy, gold nanoparticles (GNPs) are regarded as a promising radiosensitizer candidate. Several studies have revealed a dose enhancement by GNPs in X-ray and even proton irradiation. However, these studies have been limited to the depth direction. The dose distribution in both depth and lateral directions is crucial to evaluate the full radio sensitizing effect. The purpose of this study is to estimate the dose distribution around a GNP in terms of ejected electrons. Methods: The Geant4 Monte Carlo simulation toolkit was used to evaluate the energy deposition of electrons produced by a GNP. A $20 \mathrm{~nm}$ diameter spherical GNP was located in a water box and proton beams were incident unidirectionally. The energy deposition and location of produced electrons were tallied by $5 \mathrm{~nm}$ width water slabs at a variety of depths behind the GNP. The radial dose distribution was obtained in each slab. Results: The largest radial dose was observed in the slab closest to the GNP. At the slabs deeper than $90 \mathrm{~nm}$, the dose in the radial direction within $10 \mathrm{~nm}$ from the beam direction was found to be smaller than that without GNP. This is because the presence of a GNP decreases the dose behind the GNP, forming a dose shadow. The dose enhancement both in depth and lateral directions was shown in surrounding areas. The area of distribution became larger as the absorbed dose decreased. Conclusion: The dose distribution around a GNP was estimated by a simulation study. The dose enhancement was observed in both the lateral and depth directions. This study will enable us to make use of GNPs as a radiosensitizer in proton therapy.
\end{abstract}

\section{Keywords}

Gold Nanoparticle, Proton Therapy, Geant4, Radial Dose Distribution 


\section{Introduction}

Radiation therapy has been widely conducted as an effective means to eradicate tumor tissues. To treat refractory cancer tissues, however, dose should be more concentrated than to other tumor tissues. For enhancing the radiation effects on such obstinate tissue, Gold nanoparticles (GNPs) have been regarded as a promising candidate radiosensitizer [1]-[3]. GNPs are functionalized with proteins such as antibodies or peptides so that they can target tumor cells [4] [5]. Inside the tumor cell exposed to X-rays, GNPs produce secondary electrons and enhance the dose due to the high atomic number of gold $(\mathrm{Z}=79)$ [6]-[8].

Meanwhile, particle therapy, including proton therapy, is known to provide a desirable dose distribution in which a high dose region is formed at a depth referred to as the Bragg Peak [9]. In spite of this physical characteristic, there have been few published investigations concerning the dose enhancement by GNPs with proton irradiation. In vivo and in vitro studies have shown that GNPs exhibit a remarkable radiosensitization effect even with proton irradiation [10]-[12]. From a theoretical point of view, the dose enhancement by secondary electrons ejected from GNPs exposed to protons decreases more rapidly with distance from the GNP surface compared to photon irradiation because the energy transfer to electrons from a proton is smaller than that from a photon (X-ray or $\gamma$-ray) [13] [14].

In order to realize the application of GNPs as a clinical radiosensitizer in proton therapy, it is essential to know the dose distribution around GNPs in detail. For both photon and proton beams, several reports have revealed the dose enhancement in the depth direction [7] [13]-[15]. However, as far as we know, no studies considering the radial dose distribution of electrons ejected from GNPs in proton irradiation have been reported. In this article, we present calculations of the radial dose distribution caused by secondary electrons from a GNP using the Geant4 Monte Carlo simulation toolkit [16]. It is shown that GNPs enhance the dose not only in the depth direction, but also in the radial direction.

\section{Methods and Materials}

The radial dose distribution of electrons produced by physical reactions between proton beams and a GNP was calculated by a simulation with the Geant4 Monte Carlo toolkit (version 4.10.0). The G4EmLivermorePhysics list was used to track low energy electrons down to $10 \mathrm{eV}$. The step length was set to $0.1 \mathrm{~nm}$.

A spherical $20 \mathrm{~nm}$ diameter GNP was located at $0.5 \mu \mathrm{m}$ depth in a liquid water box ( $3 \mu \mathrm{m} \times 1 \mu \mathrm{m} \times 1 \mu \mathrm{m})$ as shown in Figure 1. The GNP was unidirectionally irradiated by $10^{6}$ protons. Considering the clinical application, GNPs should be irradiated by a relatively low energy proton beam so that the Bragg Peak can be formed at the location of the GNPs inside tumors to maximize the dose enhancement effect. For this reason, we chose 0.7 $\mathrm{MeV}$ protons as the incident beam which formed the Bragg peak at about $0.5 \mu \mathrm{m}$ depth in the water box. Multiple water slabs with $5 \mathrm{~nm}$ thickness $(5 \mathrm{~nm} \times 1 \mu \mathrm{m} \times 1 \mu \mathrm{m}$ ) were positioned at intervals of $15 \mathrm{~nm}$ from 0 to 500 $\mathrm{nm}$ and at intervals of $100 \mathrm{~nm}$ from $500 \mathrm{~nm}$ to $2.5 \mu \mathrm{m}$ behind the GNP to tally the energy deposition and position of electrons.

Next, from the energy deposition and position data, the radial dose distribution was plotted as a function of the distance from the proton beam direction in each slab. To evaluate the enhancement effect, the energy deposition without the GNP was subtracted from the energy deposition in the presence of the GNP.

Finally, the absorbed dose distribution by electrons was evaluated from the relationship between the GNPslab distance and proton beam direction-electron distance. Here, the dose distribution in each slab was obtained by averaging the dose from $-90 \%$ to $+110 \%$.

\section{Results}

Radial dose distributions at locations for 3 slabs are shown in Figure 2. The dose in the figure represents the subtracted dose $\left(D_{\text {sub }}\right)$ in which the dose with GNP $\left(D_{\text {with GNP }}\right)$ is subtracted by the dose without GNP $\left(D_{\text {without GNP }}\right)$. It was shown that the doses at 0 and $15 \mathrm{~nm}$ from the GNP surface decrease similarly after $10 \mathrm{~nm}$, while the dose at $90 \mathrm{~nm}$ from the GNP surface is slightly lower than that without GNP at up to about $2 \mathrm{~nm}$ from the proton beam direction (beam axis). This latter minus region is referred to as a "dose shadow" that is caused by proton collision with the GNP.

Figure 3 shows the dose distribution as a function of depth and radial distance from the GNP center. In this figure, the proton beam is incident on the GNP sphere from the left. From 1 to 1000 [ $\mu \mathrm{Gy} /$ particle], the distribution area spreads in the lateral direction as well as in the depth direction as the dose value becomes smaller. 


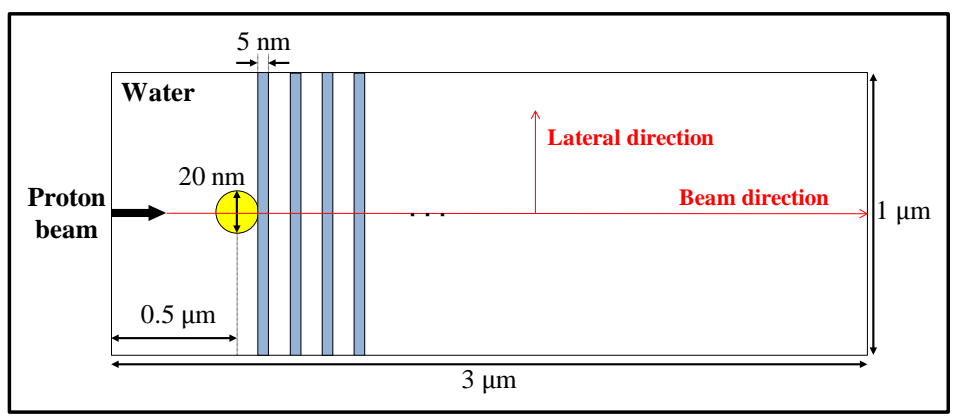

Figure 1. Geometry of the Geant4 simulation set up. A $20 \mathrm{~nm}$ diameter gold nanoparticle (GNP) is located at $0.5 \mu \mathrm{m}$ depth in a liquid water phantom. The GNP is irradiated by a proton beam unidirectionally from the left. Five nanometer thickness water slabs are positioned behind the nanoparticle to tally the energy deposition and location of electrons. Tally slabs are positioned at intervals of $15 \mathrm{~nm}$ from 0 to $500 \mathrm{~nm}$ and $100 \mathrm{~nm}$ from $500 \mathrm{~nm}$ to $2.5 \mu \mathrm{m}$.

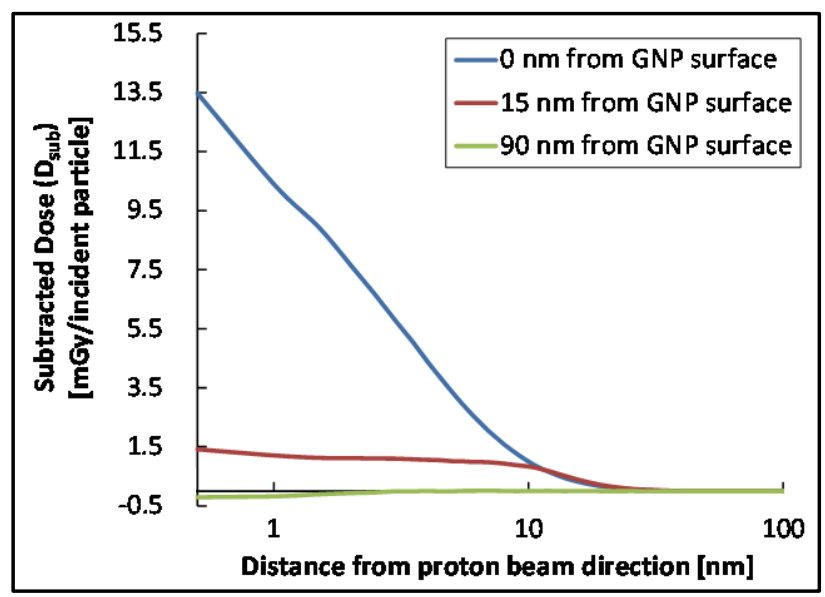

Figure 2. Radial dose distribution $\left(D_{\text {sub }}=D_{\text {with GNP }}-D_{\text {without GNP }}\right)$ of electrons ejected from GNP at 3 slabs. The blue line represents $0 \mathrm{~nm}$, the red line $15 \mathrm{~nm}$, and the green line $90 \mathrm{~nm}$ from GNP surface. The dose represents the value divided by the number of incident protons $\left(10^{6}\right)$.

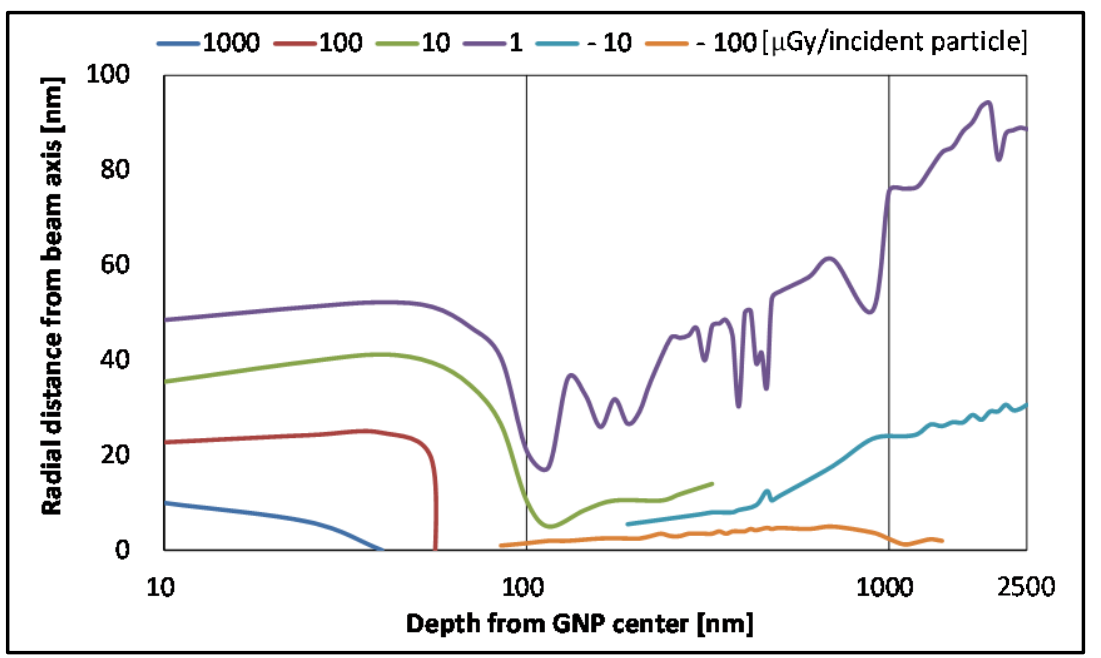

Figure 3. Dose distribution around a GNP. Each line represents 1000, 100, 10, 1, -10, and -100 [ $\mu \mathrm{G} y /$ incident particle]. Since the dose without GNP is subtracted from the dose in the presence of GNPs, the dose presented in the figure can become lower than zero, which is shown at depths larger than $90 \mathrm{~nm}$ from the GNP center. 
However, the region where the dose became less than $0[\mu \mathrm{Gy} /$ particle] appears in a narrow range after $90 \mathrm{~nm}$ from the GNP center. As an attempt to assume the effective volume being bounded by $D_{\text {sub }}=1$ [ $\mu \mathrm{Gy} /$ particle] and simplified as a cuboid with a volume of $2.5 \times 10^{-14}\left[\mathrm{~cm}^{3}\right](=100 \mathrm{~nm} \times 100 \mathrm{~nm} \times 2.5 \mu \mathrm{m})$, the effect around the GNP is not insignificant when the density of GNPs is larger than $4 \times 10^{13}\left[1 / \mathrm{cm}^{3}\right]\left(=1 /\left(2.5 \times 10^{-14}\right)\right)$.

\section{Discussion}

In this study, we have shown that a GNP irradiated by protons enhances the dose not only in the depth direction but also in the radial direction from the proton beam axis. The dose distribution suggests that the effect (for 1 [ $\mu \mathrm{Gy} /$ incident proton] of $\mathrm{D}_{\text {sub }}$ ) extends over several micrometers in the depth direction and several tens of nanometers in the radial direction.

It is well known that DNA double-strand breaks (DSBs) are a major cause of cell death [17]. Lin et al. reported that the dose by proton irradiation decreases rapidly at around $10 \mu \mathrm{m}$ from the GNPs surface, which is much shorter in length than that by photon irradiation [14]. This indicates the importance of effective cellular uptake of drugs that contain GNPs near the cell nucleus of $10 \mu \mathrm{m}$ in diameter. As shown in this study, the dose by proton is spread around a GNP in multiple directions, which can be utilized to enhance the treatment effect [18].

The dose enhancement ratio (DER) is often used to evaluate the effectiveness of radiosensitizers. However, DER is inappropriate in this study because the ratio of $\mathrm{D}_{\text {with GNP }}$ to $\mathrm{D}_{\text {without GNP }}$ can be quite large (sometimes infinity) at some local points. That is why we subtracted $D_{\text {without } G N P}$ from $D_{\text {with }}$ GNP.

There are a number of limitations with the present study. A fundamental physical dose distribution in a simple geometry by means of a Geant 4 Monte Carlo simulation is presented. Although more specific study is necessary in terms of the simulation geometry and proton beam energy, the result of dose enhancement around a GNP in this study will help us estimate the effective length and to achieve an appropriate drug concentration.

\section{Conclusion}

In this study, we investigated the effective distance of the dose enhancement around a GNP exposed to a proton beam by a Monte Carlo simulation. The dose enhancement was confirmed in the region within $2.5 \mu \mathrm{m}$ in depth direction and within $100 \mathrm{~nm}$ in lateral direction relative to the beam axis. A dose shadow was also observed at the depth from $90 \mathrm{~nm}$ to $2.5 \mu \mathrm{m}$ distal to the GNP. The results will enable us to project the radiosensitizing effect in clinical applications of proton therapy.

\section{Acknowledgements}

This work was supported by JSPS KAKENHI Grant Number 26461876.

\section{References}

[1] Hainfeld, J.F., Dilmanian, F.A., Slatkin, D.N. and Smilowitz, H.M. (2008) Radiotherapy Enhancement with Gold Nanoparticles. Journal of Pharmacy and Pharmacology, 60, 977-985. http://dx.doi.org/10.1211/jpp.60.8.0005

[2] Chithrani, D.B., Jelveh, S., Jalali, F., van Prooijen, M., Allen, C., et al. (2010) Gold Nanoparticles as Radiation Sensitizers in Cancer Therapy. Radiation Research, 173, 719-728. http://dx.doi.org/10.1667/RR1984.1

[3] Jain, S., Coulter, J.A., Hounsell, A.R., Butterworth, K.T., McMahon, S.J., et al. (2011) Cell-Specific Radiosensitization by Gold Nanoparticles at Megavoltage Radiation Energies. International Journal of Radiation Oncology • Biology - Physics, 79, 531-539. http://dx.doi.org/10.1016/j.ijrobp.2010.08.044

[4] Nativo, P., Prior, I.A. and Brust, M. (2008) Uptake and Intracellular Fate of Surface-Modified Gold Nanoparticles. ACS Nano, 2, 1639-1644. http://dx.doi.org/10.1021/nn800330a

[5] Jiang, W., Kim, B.Y., Rutka, J.T. and Chan, W.C. (2008) Nanoparticle-Mediated Cellular Response Is Size-Dependent. Nature Nanotechnology, 3, 145-150. http://dx.doi.org/10.1038/nnano.2008.30

[6] Cho, S.H. (2005) Estimation of Tumour Dose Enhancement Due to Gold Nanoparticles during Typical Radiation Treatments: A Preliminary Monte Carlo Study. Physics in Medicine and Biology, 50, N163.

[7] McMahon, S.J., Hyland, W.B., Muir, M.F., Coulter, J.A., Jain, S., et al. (2011) Nanodosimetric Effects of Gold Nanoparticles in Megavoltage Radiation Therapy. Radiotherapy and Oncology, 100, 412-416. http://dx.doi.org/10.1016/j.radonc.2011.08.026 
[8] Chow, J.C., Leung, M.K., Fahey, S., Chithrani, D.B. and Jaffray, D.A. (2012) Monte Carlo Simulation on Low-Energy Electrons from Gold Nanoparticle in Radiotherapy. Journal of Physics: Conference Series, 341, Article ID: 012012.

[9] Miralbell, R., Lomax, A., Cella, L. and Schneider, U. (2002) Potential Reduction of the Incidence of Radiation-Induced Second Cancers by Using Proton Beams in the Treatment of Pediatric Tumors. International Journal of Radiation Oncology • Biology • Physics, 54, 824-829. http://dx.doi.org/10.1016/S0360-3016(02)02982-6

[10] Liu, C.J., Wang, C.H., Chen, S.T., Chen, H.H., Leng, W.H., et al. (2010) Enhancement of Cell Radiation Sensitivity by Pegylated Gold Nanoparticles. Physics in Medicine and Biology, 55, 931. http://dx.doi.org/10.1088/0031-9155/55/4/002

[11] Polf, J.C., Bronk, L.F., Driessen, W.H., Arap, W., Pasqualini, R., et al. (2011) Enhanced Relative Biological Effectiveness of Proton Radiotherapy in Tumor Cells with Internalized Gold Nanoparticles. Applied Physics Letters, 98, Article ID: 193702. http://dx.doi.org/10.1063/1.3589914

[12] Kim, J.K., Seo, S.J., Kim, H.T., Kim, K.H., Chung, M.H., et al. (2012) Enhanced Proton Treatment in Mouse Tumors through Proton Irradiated Nanoradiator Effects on Metallic Nanoparticles. Physics in Medicine and Biology, 57, 8309. http://dx.doi.org/10.1088/0031-9155/57/24/8309

[13] Gao, J. and Zheng, Y. (2014) Monte Carlo Study of Secondary Electron Production from Gold Nanoparticle in Proton Beam Irradiation. International Journal of Cancer Therapy and Oncology, 2, Article ID: 02025. http://dx.doi.org/10.14319/ijcto.0202.5

[14] Lin, Y., McMahon, S.J., Scarpelli, M., Paganetti, H. and Schuemann, J. (2014) Comparing Gold Nano-Particle Enhanced Radiotherapy with Protons, Megavoltage Photons and Kilovoltage Photons: A Monte Carlo Simulation. Physics in Medicine and Biology, 59, 7675-7689. http://dx.doi.org/10.1088/0031-9155/59/24/7675

[15] McMahon, S.J., Hyland, W.B., Muir, M.F., Coulter, J.A., Jain, S., et al. (2011) Biological Consequences of Nanoscale Energy Deposition near Irradiated Heavy Atom Nanoparticles. Scientific Reports, 1, Article Number: 18.

[16] Agostinelli, S., Allison, J., Amako, K.A., Apostolakis, J., Araujo, H., et al. (2003) GEANT4—A Simulation Toolkit. Nuclear Instruments and Methods in Physics Research Section A: Accelerators, Spectrometers, Detectors and Associated Equipment, 506, 250-303. http://dx.doi.org/10.1016/S0168-9002(03)01368-8

[17] Khanna, K.K. and Jackson, S.P. (2001) DNA Double-Strand Breaks: Signaling, Repair and the Cancer Connection. Nature Genetics, 27, 247-254. http://dx.doi.org/10.1038/85798

[18] Douglass, M., Bezak, E. and Penfold, S. (2013) Monte Carlo Investigation of the Increased Radiation Deposition Due to Gold Nanoparticles Using Kilovoltage and Megavoltage Photons in a 3D Randomized Cell Model. Medical Physics, 40, Article ID: 071710. http://dx.doi.org/10.1118/1.4808150 
Scientific Research Publishing (SCIRP) is one of the largest Open Access journal publishers. It is currently publishing more than 200 open access, online, peer-reviewed journals covering a wide range of academic disciplines. SCIRP serves the worldwide academic communities and contributes to the progress and application of science with its publication.

Other selected journals from SCIRP are listed as below. Submit your manuscript to us via either submit@scirp.org or Online Submission Portal.
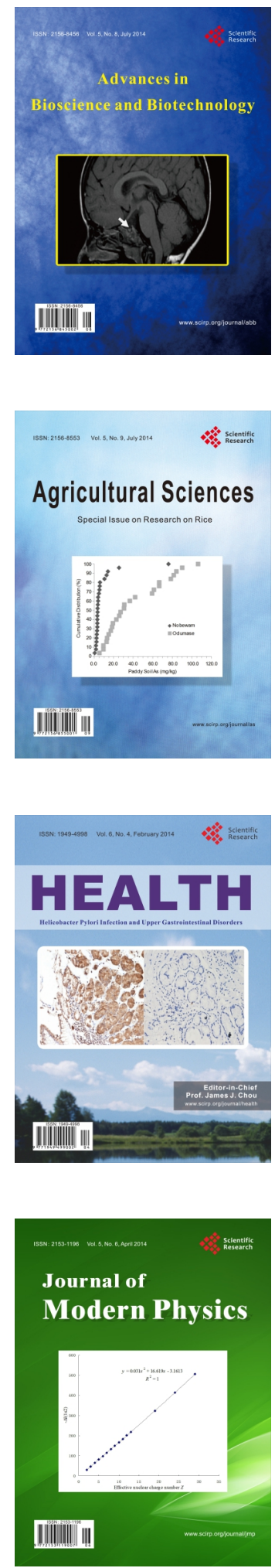
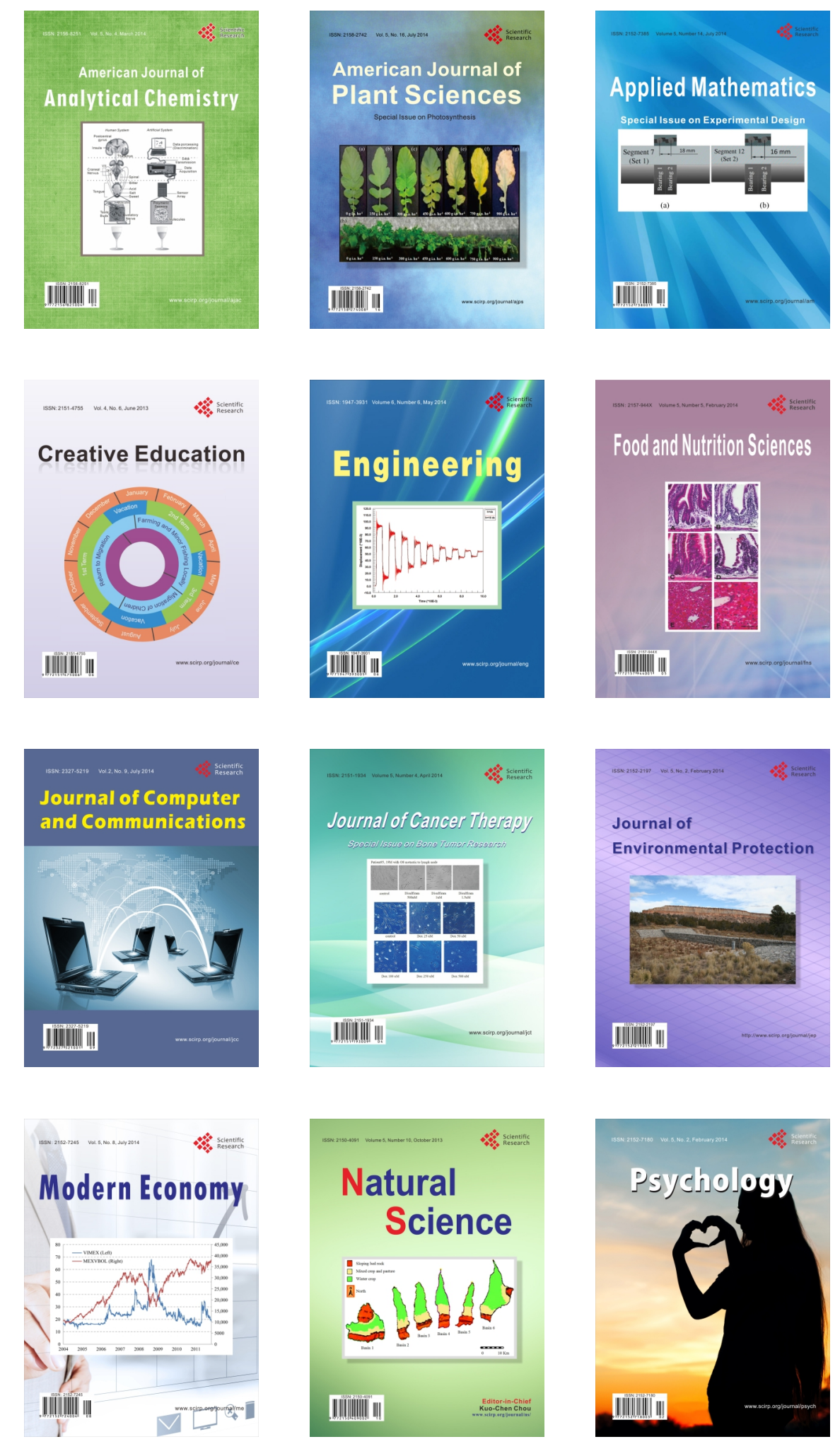